\title{
Challenges of Preoperative Diagnosis and Management of Scalene Intramuscular Angioma
}

\author{
Lei Zhang ${ }^{1 *}$, Beverly Wang ${ }^{2}, \&$ Julie Goddard ${ }^{3}$ \\ ${ }^{1}$ University of California at Los Angeles, USA \\ ${ }^{2}$ University of California at Irvine, department of pathology, USA \\ ${ }^{3}$ University of California at Irvine, head and neck surgery, USA \\ Correspondence: Lei Zhang, University of California at Los Angeles, USA. E-mail: lei_248@hotmail.com
}

Received: November 17, 2015 Accepted: December 13, 2015 Online Published: December 18, 2015

doi: $10.5539 /$ cco.v5n $1 \mathrm{p} 20$

URL: http://dx.doi.org/10.5539/cco.v5n1p20

\begin{abstract}
Intramuscular angioma of scalene muscle is rare with only five cases reported so far. Four of them have not been suspected before surgery; one was diagnosed preoperatively by core biopsy. Preoperative diagnosis is important for management. Awareness of cytologic features could help preoperative diagnosis when need of ruling out malignancy and coagulopathy make fine needle aspiration a choice. We herein demonstrate a new case of a 27 year old male with history of hepatocellular carcinoma, who presented with a $6 \mathrm{~cm}$ left supraclavicular mass. The fine needle aspiration was paucicellular; however, the bland ovoid to spindle cells with a whirling and luminal arrangement in the background of blood, fatty drops and degenerate muscle are suggestive of intramuscular angioma. The magnetic resonance imagines (MRI) demonstrate a T1 isointense and T2 hyperintense ill-defined lesion splaying anterior and mid scalene muscles with subtle vascular voids at periphery. These features in combination with cytology findings indicate intramuscular angioma. The pre-operative findings are correlated to the histologic picture of mixed capillaries and varying sized venues intervening with fatty tissue and atrophic muscle. Intraoperatively, the mass is adjacent to the brachial plexus rootlets, interdigitating with the scalene muscle and pushing the carotid sheath, left subclavicular artery and vein aside. Following embolization, the mass is resected with minimal bleeding. Our case suggests that scalene intramuscular angioma can be successfully managed by surgery after embolization; preoperative diagnosis rendered by cytologic features and imaging characters would aid the planning of surgery.
\end{abstract}

\section{Introduction}

Intramuscular angioma is relatively rare accounting for less than $1 \%$ of all benign soft tissue tumors (Weiss, Goldblum, Murpey, \& Kransdorf, 2008), despite the fact that it is one of the most frequent deep seated soft tissue tumors (Calonje, 2013). The head and neck location is uncommon representing $15 \%$ of intramuscular angioma, with subsites in a decreasing order of masseter muscle, trapezius, and others such as temporalis, periorbital muscles, mylohyoid, sternocleidomastoid and buccinators (Rossiter, Hendrix, Tom, \& Potsic, 1993; Moumoulidis, Durvasula, \& Jani, 2007; Wierzbicki, Henderson, Scarborough, Bush, Reith, \& Clugston, 2013). Among the over 80 head and neck intramuscular angioma cases reported, there are only five located in scalene musculature and $80 \%(4 / 5)$ of them have not been suspected preoperatively (Van Abel, Carlson, Janus, Torres-Mora, Moore, Olsen, \& Link, 2013; Ferlito \& Gale, 1980; Scott, 1957; Cho, Cha, \& Sung, 2015). Of the four unexpected cases, two were operated with presumptive diagnosis of paraganglioma; the other two had no preliminary diagnosis and significant bleeding occurred during surgery in one case (Van Abel, Carlson, Janus, Torres-Mora, Moore, Olsen, \& Link, 2013; Ferlito \& Gale, 1980; Scott, 1957). Only one case was diagnosed preoperatively by core biopsy (Cho, Cha, \& Sung, 2015). The correct categorization before surgery is import for management planning as the approach of intramuscular angioma is individualized with choices of observation, surgery or combined therapy (Wierzbicki, Henderson, Scarborough, Bush, Reith, \& Clugston, 2013). In general, the diagnosis of intramuscular angioma was not apparent most of time until histologic examination was performed (Moumoulidis, Durvasula, \& Jani, 2007; Cho, Cha, \& Sung, 2015). However, there are situations where fine needle aspiration (FNA) is the choice and preoperative interpretation is based on cytomorphology. FNA is usually considered when there is a concern for metastasis and the patient is at risk for bleeding. It provides the benefits of sampling a larger area by changing angles of a needle and minimizing bleeding through 
use of small gauge needles. Overall, deeply sit angioma is a challenge for preoperative diagnosis due to: 1) absence of specific clinical signs such as pulsation or skin bruise (Weiss, Goldblum, Murpey, \& Kransdorf, 2008); 2) overlapping imaging findings with other soft tissue tumors and variant imagines within intramuscular angioma (Weiss, Goldblum, Murpey, \& Kransdorf, 2008; Wierzbicki, Henderson, Scarborough, Bush, Reith, \& Clugston, 2013; Van Abel, Carlson, Janus, Torres-Mora, Moore, Olsen, \& Link, 2013); 3) scant cellularity on fine needle aspiration (FNA) (Moumoulidis, Durvasula, \& Jani, 2007); 4) and rarity of the disease. We present a case of intramuscular angioma of scalene muscle in a 27 year old man who had a history of hepatocellular carcinoma. The diagnosis was suspected preoperatively through fine needle aspiration and imagines studies. Embolization prior to surgery and careful dissection led to minimal bleeding and no palsy complication.

\section{Clinical History}

A 27-year old man reported a slow growing painless left supraclavicular mass for 3 months, reaching up to $6 \mathrm{~cm}$ in size. The patient is a liver transplant candidate with pathology proven hepatocellular carcinoma, history of hepatitis C, cirrhosis, and esophageal varices status post banding for bleed. The CBC shows $\mathrm{Hb} 9.3 \mathrm{~g} / \mathrm{dl}$ (reference: $13.5-16.9$ ), platelet $73 \times 10^{3} / \mathrm{MCL}$ (reference: $150-400$ ). The PTT is 38.5 SEC (reference: $24.0-32.6$ ); prothrombin time is 13.3 SEC (reference: 9.8-11.4). The alpha-fetoprotein is $20.3 \mathrm{ng} / \mathrm{ml}$ (reference: 0-5.5). Concerning for metastasis, ultrasound guided FNA was performed. Ten passes were obtained using 25 and 18 gauge needles. Incisional biopsy in the operating room led to brisk bleeding controlled with clips, biopolar electrocautery and surgicel. The mass was felt to be a vascular lesion difficult for delivery from the deeper structures especially as its inferior aspect. Considering that the patient with a liver transplant should potentially have a normal life span, a complete resection of curable disease is preferred. The surgical team proceeded to a radical excision another day after embolization was performed and neurosurgery, cardiothoracic surgery and intraoperative neuro monitoring for the brachial plexus were all available. During the resection, the mass was noted to be between the anterior and middle scalene muscles, in close proximity to the apex of lung, just medial to the brachial plexus rootlets, pushing the left internal jugular vein /carotid artery medially and left subclavicular artery and vein inferiorly and anteriorly. The surgery went well with minimal bleeding and no debilitating complication. The patient has been followed up and symptom free for 6 months.

\section{Pathologic Findings}

Ten passes of FNAs show paucicellular aspirations composed mostly of blood in the background of fatty droplets and rare clusters of striated degenerate muscles. There are whirling spindle and ovoid cells with vague lumen formation which are different from random stroma. Those cells are endothelial appearing with minimal atypia. There is no prominent nucleolus to suggest epithelioid endothelial cells or waving nuclei to indicate neurogenic lesion. There is no cytoplasmic granule which could be seen in paraganglioma. The stroma is not myxoid either. Occasional multinucleated cells recognized as macrophages or fused endothelial cells are present. No significant lymphocytes, plasma cells, eosinophils, or any lipoblast is identified (Figure 1 A-B). No mitosis is seen. Cell block demonstrates one dilated irregularly shaped vein and numerous red blood cells. The cytologic diagnosis is: blood components and rare bland spindle cells, negative for metastatic disease or malignancy; considering hematoma and angioma.

The frozen sections of the incisional biopsy show venous vasculature interdigitating with mature adipose tissue and muscles. The excision shows an ill-defined, yellow to white tan solid mass with hemorrhagic clefts (Figure 1 D). Sections reveal ectatic and compressed venues varying in size and wall thickness (Figure $1 \mathrm{E}$ ). Foci of capillary lobules are also present (Figure $1 \mathrm{E}-\mathrm{F}$ ). The composite cells are diffusely positive for endothelial markers CD31, CD34, smooth muscle actin (SMA); focal rarely positive for D2-40; negative for HHV-8. The vascular lesion is partially replaced by mature fatty tissue. Infiltrative growth led to muscle degeneration (Figure E-F). The lack of prominent nucleoli rules out epithelioid angioma or epithelioid hemangioendothelioma (the intermediate grade vascular tumors). The absence of anastomosing vessels, mitotic activity or cytologic atypia excludes angiosarcoma. The negativity for HHV-8 and absence of spindle cell lined interdigitating space filled by lymphocytes/plasma cells make Kaposi sarcoma unlikely. The histologic diagnosis in pathology is intramuscular angioma.

\section{Radiology Images}

The lesion is hyperechogenic on ultrasound. The MR appearance of the left supraclavicular mass in Figure $1 \mathrm{G}$ $\& \mathrm{H}$ is of iso-intensity (similar to that of muscle) on T1-weighted images, and high signal intensity (greater than or equal to that of subcutaneous fat) on T2-weighted images, which reflects the preponderance of water contents or fluid-filled cystic spaces. However, the serpiginous flow voids (Figure $1 \mathrm{H}$ ) are vague and there is no hypo-dense nidus internally to suggest high flow vascular lesion. The focal high peripheral signal density on 
both T1 and T2 phases (black arrows) suggest fat in the mass. The CT image confirms no bone involvement or calcification/phlebolith (Figure $1 \mathrm{I}$ ). The extent of the lesion is also determined by magnetic resonance angiography (MRA) which shows displacement of adjacent carotid artery, subclavian artery and vein but without significant tumor vasculature (Figure $1 \mathrm{~J}$ ).

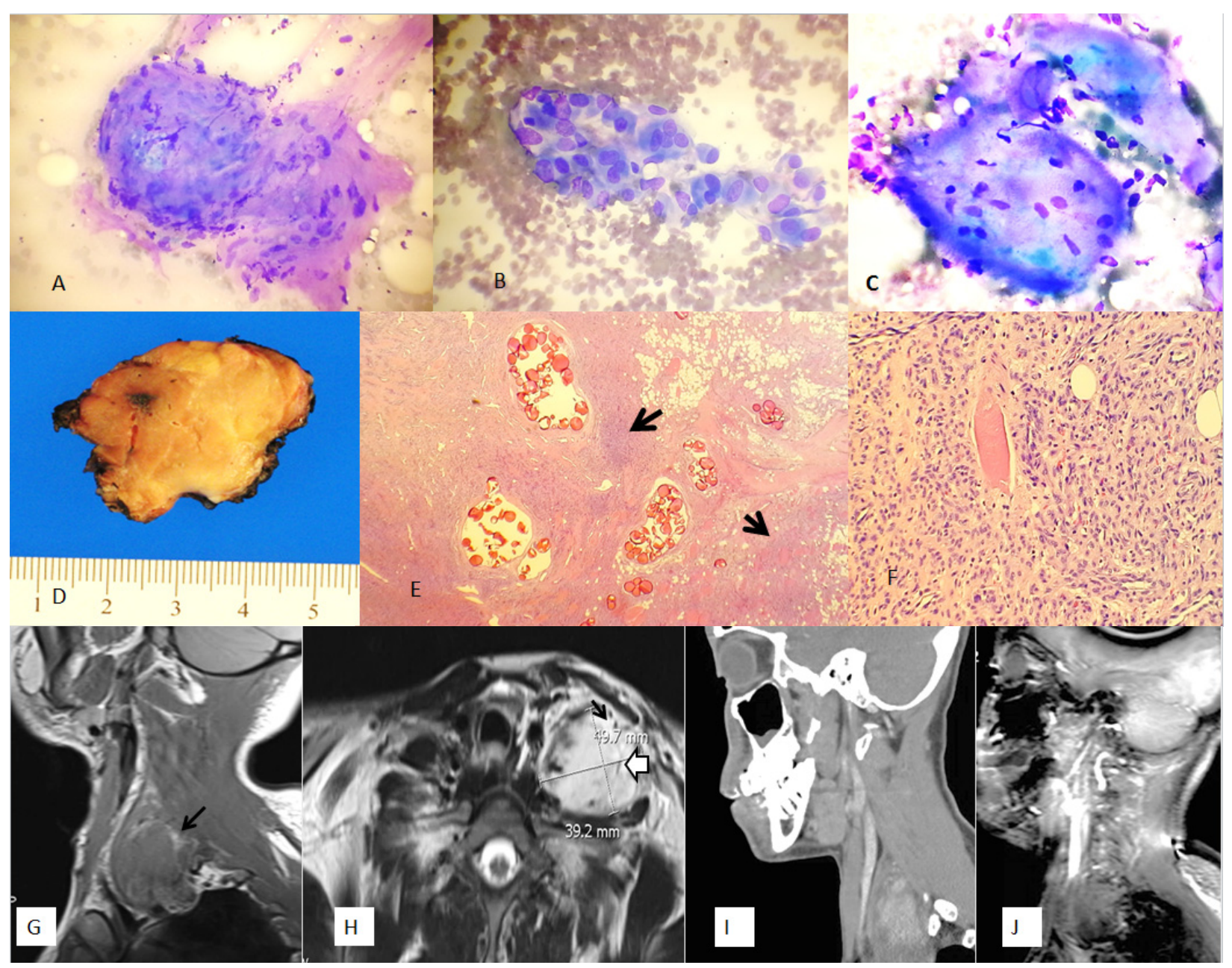

Figure 1. Pathologic findings and radiology images

A-C: Fine needle aspiration of left neck mass. Immediate Diff Quik stains of smears show spindle cells arranged in a tightly well formed, three-dimensional swirling pattern with associated fatty droplets (A). Minimally atypical ovoid cells with vague membrane groove around small lumens are present (B). Background degenerated muscle cells with striate are identified (C).

D-F: Histology of resection. The mass is solid and yellowish with vascular clefts and focal hemorrhage grossly (D). The tumor dissects into muscle and is composed of large (cavernous) and small vessels (capillaries) admixed with fatty tissue (E). The representative high power view of capillary angioma area (arrow in E) is shown in $\mathrm{F}$.

G-J: Imagines. G (MRI, T1 phase): left supraclavicular mass $(6 \mathrm{~cm}$ superior-inferior) with $\mathrm{T} 1$ isointense enhancement and focal hyperintense area at periphery (arrow). H (MRI, T2 phase): same mass with avid T2 central enhancement, subtle vascular flow voids (open arrow) and peripheral hyperdense area suggestive of fat (black arrow). I (CT): mass lesion with attenuation similar to that of muscle, no bone involvement or calcification. J (MRA): mass with associated inferior displacement of the left subclavian artery, medially displacement of left carotid artery and anterior displacement of the left subclavian vein, which otherwise demonstrates preserved flow voids. 
Overall, the image findings do not agree with adipose tumor which usually is bright at both T1 and T2 MRI; they do not seem to be metastatic lesion either in which loss of signal intensity would be expected. The differentiations of the high water content mass in this supraclavicular location are either vascular (non-arterial type), neuroendocrine or myxoid tumor.

\section{Discussion}

MRI is the choice of image to explore intramuscular angioma, which typically reveals a T1 low density and T2 high density lesion (Weiss, Goldblum, Murpey, \& Kransdorf, 2008; Calonje, 2013; Wierzbicki, Henderson, Scarborough, Bush, Reith, \& Clugston, 2013; Van Abel, Carlson, Janus, Torres-Mora, Moore, Olsen, \& Link, 2013). Although these features are characteristic, diagnostic specificity is increased if serpiginous flow voids and hypo-dense nidus representing high flow lesion are present (Weiss, Goldblum, Murpey, \& Kransdorf, 2008; Calonje, 2013; Vilanova et al., 2004). MRI can suggest intramuscular angioma in a typical location and presentation such as painful mass in the extremity muscle; but FNA or biopsy may still be required in a case with uncommon location or unusual presentation (Moumoulidis, Durvasula, \& Jani, 2007; Van Abel, Carlson, Janus, Torres-Mora, Moore, Olsen, \& Link, 2013; Layfield, Mooney, \& Dodd, 1998).

The FNA shows all the diagnostic elements for intramuscular angioma although paucicellular, e.g., the bland ovoid to spindle cells with a whirling and luminal arrangement in the background of blood, fatty drops and degenerate muscle (Layfield, Mooney, \& Dodd, 1998; DeMay, 2012).

Intramuscular angioma occurs mostly in adolescents and young adults, is likely congenital malformations rather than a consequence to trauma. It has been radiographically categorized into high- and low- flow based on hemodynamic characters (Wierzbicki, Henderson, Scarborough, Bush, Reith, \& Clugston, 2013; Vilanova et al., 2004); and histologically classified into small (capillary), large (cavernous) and mixed types based on size of vessels (Calonje, 2013; Allen \& Enzinger, 1972). The large vessel type with artery component (arterial malformation, arteriovenous malformation/fistula) is associated with high flow; while the venous or lymphatic or mix of both is of low flow. Capillary type can be both high and low flow based on feeding vessels, artery or vein. Grossly, the small vessel type appears more solid than large vessel type; and this tissue texture is pertinent to MRI density. Of note, intramuscular angiomas in the scalene vasculature are mostly mixed large and small vessel type $(5 / 6,83 \%)$ with one case reported to be capillary type (1/6, 17\%) (Van Abel, Carlson, Janus, Torres-Mora, Moore, Olsen \& Link, 2013; Ferlito \& Gale, 1980; Scott, 1957; Cho, Cha, \& Sung, 2015). This is in contrast to that in sternocleidomastoid muscle which $60 \%(3 / 5)$ of them are large vessel (cavernous) type, $20 \%(1 / 5)$ is small vessel (capillary) type and 20\% (1/5) is mixed type (Moumoulidis, Durvasula, \& Jani, 2007).

After the diagnosis, the management is mostly individualized varying from conservative follow up with activity modification, systemic corticosteroids, radiation, embolization, sclerotherapy and surgical excision (Wierzbicki, Henderson, Scarborough, Bush, Reith, \& Clugston, 2013). The judgement is based on the characters of the lesion and patient functional malformation. Because of the self-limited nature and involution of most angiomas, surgical intervention is not needed in most cases (Wierzbicki, Henderson, Scarborough, Bush, Reith, \& Clugston, 2013). Indications for surgery include rapid tumor growth, suspicion of malignancy, intractable pain, thrombocytopenia, local skin necrosis, cosmetic or impairment of function. Surgical intervention is the choice for our case because of the concern for metastasis, growing tumor reaching a big size and proximity to nerve and vessels.

Hemorrhage is the most common complication for surgery occurring in $20 \%$ of cases followed by transient nerve palsy (Rossiter, Hendrix, Tom, \& Potsic, 1993; Scott, 1957). This has been controlled by pre-operative embolization in our case. Embolization has also been shown to decrease risk of recurrence in operable cases and control pain in unresectable lesions (Rossiter, Hendrix, Tom, \& Potsic, 1993; Bowman, Johnson, McKusick, Gloviczki, \& Driscoll, 2013). Inadequate excision has the greatest risk for recurrence; while adequate resection may pose danger of debilitating complications. Therefore, the rate of post-surgery recurrence varies from $18 \%$ to 61\% (Wierzbicki, Henderson, Scarborough, Bush, Reith, \& Clugston, 2013).

\section{Summary}

In summary, we present this case for awareness of the rare presentation of intramuscular angioma in the scalene muscles. Cytology and MRI combined can make the pre-operative diagnosis. Surgery together with embolization seems suitable for big intramuscular angioma with complex anatomy. 


\section{Conflict of Interest}

The authors declare that they have no competing interests.

\section{Author's Contributions}

All authors contributed to diagnosis, data collection, literature search and discussion. JD performed the surgery and collected the specimens. BW and LZ made the cytohistologic diagnosis. LZ assembled the data and wrote the manuscript. All authors approved the final writing.

\section{References}

Allen, P. W., \& Enzinger, F. M. (1972). Hemangioma of skeletal muscle. An analysis of 89 cases. Cancer, 29(1), 8-22. http://dx.doi.org/10.1002/1097-0142(197201)29:1<8::AID-CNCR2820290103>3.0.CO;2-A

Bowman, J., Johnson, J., McKusick, M., Gloviczki, P., \& Driscoll, D. (2013). Outcomes of sclerotherapy and embolization for arteriovenous and venous malformations. Semin Vasc Surg., 26(1), 48-54. http://dx.doi.org/10.1053/j.semvascsurg.2013.04.001

Calonje, J. E. (2013). Haemangioma. In C. D. M. Fletcher, J. A. Bridge, P. C. W. Hogendoorn \& F. Mertens (Eds.), WHO classification of tumors of soft tissue and bone (4th ed., pp. 138-139). IARC: Lyon. (ISBN 978-92-832-2434-1).

Cho, J. K., Cha, W., \& Sung, M. W. (2015). Intramuscular hemangioma in the anterior scalene muscle diagnosed by core needle biopsy. Clin Exp Otorhinolaryngol, 8(3), 298-301. http://dx.doi.org/10.3342/ceo.2015.8.3.298

DeMay, R. M. (2012). Vascular tumors. In I. L. Chicago (Ed.), The Art \& Science of Cytopathology (2nd ed., pp. 678-682). ASCP Press.

Ferlito, P. N., \& Gale, N. (1980). Intramuscular hemangioma of the middle scalene muscle. Acta Otorhinolaryngol Belg., 34(34), 345-349.

Layfield, L. J., Mooney, E. E., \& Dodd, L. G. (1998). Not by blood alone: diagnosis of hemangiomas by

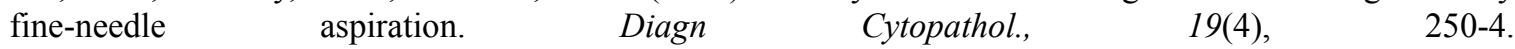
http://dx.doi.org/10.1002/(SICI)1097-0339(199810)19:4<250::AID-DC4>3.0.CO;2-E

Moumoulidis, I., Durvasula, V. S., \& Jani, P. (2007). An unusual neck lump: intramuscular haemangioma of the sternocleidomastoid muscle. Eur Arch Otorhinolaryngol, 264(10), 1257-60. http://dx.doi.org/10.1007/s00405-007-0372-3

Rossiter, J. L., Hendrix, R. A., Tom, L. W., \& Potsic, W. P. (1993). Intramuscular hemangioma of the head and neck. Otolaryngol Head Neck Surg., 108(1), 18-26. http://dx.doi.org/10.1177/019459989310800103

Scott, J. E. S. (1957). Hæmangiomata in skeletal muscle. $B r \quad J$ Surg., 44, 496-501. http://dx.doi.org/10.1002/bjs.18004418713

Van Abel, K. M., Carlson, M. L., Janus, J. R., Torres-Mora, J., Moore, E. J., Olsen, K. D., \& Link, M. J. (2013). Intramuscular hemangioma of the scalene musculature masquerading as a paraganglioma: a case series. $\mathrm{Am}$ J Otolaryngol., 34(2), 158-62. http://dx.doi.org/10.1016/j.amjoto.2012.09.009

Vilanova, J. C., Barceló, J., Smirniotopoulos, J. G., Pérez-Andrés, R., Villalón, M., Miró, J., ... Ros, P. R. (2004). Hemangioma from head to toe: MR imaging with pathologic correlation. Radiographics., 24(2), 367-85. http://dx.doi.org/10.1148/rg.242035079

Weiss, S. W., \& Goldblum, J. R. (2008). Benign tumors and tumor like lesions of blood vessels. Murpey MD and Kransdorf MJ. Radiologic evaluation of soft tissue tumors. Both In: Enzinger and Weiss's soft tissue tumors (5th ed., pp. 633-679; and 33-71). Philadelphia, PA: Mosby Elsevier (ISBN 978-0-323-04628-2)

Wierzbicki, J. M., Henderson, J. H., Scarborough, M. T., Bush, C. H., Reith, J. D., \& Clugston, J. R. (2013). Intramuscular hemangiomas. Sports Health, 5(5), 448-54. http://dx.doi.org/10.1177/1941738112470910

\section{Copyrights}

Copyright for this article is retained by the author(s), with first publication rights granted to the journal.

This is an open-access article distributed under the terms and conditions of the Creative Commons Attribution license (http://creativecommons.org/licenses/by/3.0/). 